REVISTA DE GESTTiO ESECREETRRADO

MANAGEMENT AND ADMINISTRATIVE

PROFESSIONAL REVIEW
Organização: SINSESP

Editora Científica: Dra. Cibele B. Martins

Avaliação: Double Blind Review peloSEER/OJS

Revisão: Gramatical, normativa e de formatação

Data de recebimento do artigo: $23-5-2017$

Data de aceite do artigo: $16-01-2018$

DOI: http://dx.doi.org/10.7769/gesec.v9i1.687

\title{
El presupuesto participativo de Madrid: luces y sombras
}

\section{Carmen Pineda Nebot}

Licenciada en Derecho por la Universidad Complutense de Madrid y Licenciada en Ciencia Política y de la Administración por la Universidad Autónoma de Madrid. Consultora Independiente de Administraciones Públicas. Investigadora en el Grupo de Pesquisa Controle Social do Gasto Público de la UNESP-FCL Araraquara/SP (Brasil) y en el Grupo de Pesquisa Administração Pública e Gestão Social da Universidade Federal de Viçosa (UFV, Brasil). Coordinadora de Investigación del Grupo de Trabajo Espaços Deliberativos e Governança Pública GEGOP-CLACSO 2016-2019. E-mail: carmenpinedanebot@ hotmail.com (Espanha)

\section{RESUMEN}

Curiosamente cuando en Brasil, país origen del Presupuesto Participativo, surgen muchas dificultades para su continuidad en comparación con otras innovaciones democráticas, en España comienzan a ponerse en marcha nuevas experiencias. Los cambios de gobierno, producidos después de las elecciones municipales de 2015, han provocado que muchos ayuntamientos importantes, entre ellos el de Madrid, gobernados por coaliciones de izquierda hayan decidido, como se señalaba en sus programas electorales, incorporar el Presupuesto Participativo a su proyecto de gobierno. Esta comunicación tiene como objetivo analizar, desde el conocimiento adquirido en los 25 años de funcionamiento del Presupuesto Participativo, la nueva experiencia puesta en marcha en el Ayuntamiento de Madrid. Para ello se realizará un análisis documental tanto de la experiencia en sí como de los procesos pilotos realizados durante 2015 en algunos distritos de la ciudad y se analizarán los discursos y entrevistas con miembros del gobierno municipal y de lo demás partidos presentes en el ayuntamiento.

PALABRAS ClAVE: Presupuesto Participativo. Ayuntamiento de Madrid. Tecnologías de la Información y la Comunicación. 


\title{
The participatory budget of Madrid: lights and shadows
}

\begin{abstract}
Curiously, when in Brazil, the origin of the Participatory Budget, many difficulties arise for its continuity compared to other democratic innovations, in Spain begin to start new experiences. The changes of government, produced after the municipal elections of 2015, have caused that many important municipalities, including the one of Madrid, governed by leftist coalitions have decided to incorporate the Participative Budget to their project of government. This article aims to analyze this new project and for this purpose a documentary analysis and a content analysis of the interviews carried out with some managers are carried out.
\end{abstract}

KEYWORDS: Participatory budgeting. Madrid City Council. Information and Communication Technologies.

\section{Como referenciar em APA:}

Nebot, C. P. (2018). El presupuesto participativo de Madrid: luces y sombras. R.G.Secr.,GESEC, 9(1). doi: http://dx.doi.org/10.7769/gesec.v9i1.687

\section{Como referenciar em ABNT:}

NEBOT, C. P. El presupuesto participativo de Madrid: luces y sombras. R.G.Secr.,GESEC, v. 9, n. 1, 2018. doi: http://dx.doi.org/10.7769/gesec.v9i1.687

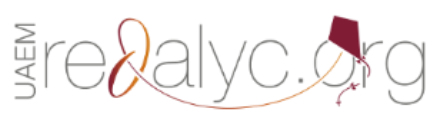
OPENACCESS 
Nebot, C. P. (2018).

\section{Introducción}

En los años 1980 y 1990 del siglo XX surge en Brasil una de las experiencias de participación ciudadana más innovadora, el Orçamento Participativo (OP), que se ha convertido en una referencia mundial. Sintomer, Herzberg y Allegretti, señalaban en 2013 la existencia de 2.788 casos distribuidos por todo el planeta, al haber sido capaz de adaptarse a las innumerables diferencias de carácter político, económico, cultural y regional presentes en el mundo (Ganuza y Baiocchi, 2012; Pateman, 2012; Sintomer et al., 2012; Porto de Oliveira, 2013; Lüchmann, 2014). Porto Alegre, capital del Estado de Rio Grande do Sul (Brasil), es la gran referencia internacional de esta metodología, al haber sido considerada por la ONU, en 1994, una de las 42 mejores prácticas de gestión urbana y por la gran difusión internacional que ha tenido.

Habiéndose mostrado el OP, en todos estos años, como una buena metodología de gestión presupuestaria para la obtención de políticas públicas más eficientes, eficaces y efectivas y para la profundización de la democracia. Su potencialidad para contribuir a cambios importantes en la relación Estado-sociedad ha sido aplaudida por actores políticos del más amplio espectro político-ideológicos y su adopción ha sido defendida por diferentes partidos y gobiernos e incentivada por organizaciones no-gubernamentales e instituciones multilaterales de desarrollo y financiación (Pires y Pineda, 2008). En la práctica, mientras tanto, el Presupuesto Participativo se enfrenta a innumerables obstáculos que dificultan enormemente la sostenibilidad de las experiencias, un claro ejemplo de ello es la pérdida de vitalidad en Brasil (Romão y da Silva, 2016). Alguno de los elementos que pueden explicar la perdida de centralidad del Presupuesto Participativo en el campo de la participación institucional en Brasil son: las dificultades de los municipios para aumentar el gasto público, la situación política y económica, los nuevos mecanismos de participación y control democráticos, los cambios en las iniciativas de la sociedad civil, la pérdida de poder del PT y la forma de gobernar del PT en los municipios (Romão y da Silva, 2016; Spada, 2014).

En Europa, en cambio, después de un periodo difícil debido, en otras razones, a la fuerte crisis económica y al triunfo político de las fuerzas conservadoras, se ha pasado a una nueva fase de auge de las experiencias. Ciudades tan importantes como Paris, Lisboa o Madrid, gobernadas por partidos de izquierda, tienen ya proyectos de presupuesto participativo, todas ellas con una característica común: el uso de las TIC. Pero no es esta la 
única diferencia entre las experiencias brasileñas y europeas, existen también diferencias socioeconómicas y políticas. En Brasil el OP surge en un momento de democratización del país y de la mano de un partido, el PT, sin experiencia de gobierno. Y con el objetivo principal de "gobernar de otra forma", es decir, de crear un nuevo pacto social entre los ciudadanos y el gobierno municipal. En cambio en España lo que se pretende es reducir la cada vez mayor desafección política, mediante la implicación de los ciudadanos.

Los factores de éxito de las experiencias de PP presenciales han estado relacionados en la literatura con las variables: voluntad política, diseño institucional y tradición asociativa (Lüchmann, 2002; Avritzer, 2003; Wampler, 2007; Borba y Lüchmann, 2007; Carlos, 2011; Horochovski y Clemente, 2012). En general, se ha considerado que cuando las tres variables se han combinado positivamente la experiencia tiene éxito, se extiende en el tiempo y es relevante para su efectividad en la elaboración del presupuesto. En el caso de los e-PP o PP mixtos, como la experiencia que vamos a analizar, al utilizarse las TIC deben tenerse también en cuenta el diseño de la página web y las herramientas digitales utilizadas.

Es importante señalar también que el presupuesto participativo sin negar el sistema representativo, el presupuesto es aprobado por los electos, incorpora a los ciudadanos de una manera directa en la elaboración y discusión de éste. Y esta incorporación la realiza desde la deliberación, elemento fundamental en este proceso. Deliberación que se convierte en un elemento motriz para muchos ciudadanos, ya que gracias a ella se escucha a otros y todos tienen la oportunidad de ponerse en el lugar de los demás. Además de permitir la inclusión heterogénea de la ciudadanía y la distribución equitativa del poder de influencia (deliberativa) entre los participantes.

Este trabajo analiza la experiencia del Presupuesto Participativo (PP) de Madrid a partir de dos de las tres variables consideradas por la literatura, durante todos estos años, factores de éxito para el éxito y continuidad de los procesos: la voluntad política y el diseño institucional (Avritzer, 2003; Wampler, 2003). Además incorporamos una nueva variable, la tecnología, debido al uso que de esta herramienta se hace tanto en esta experiencia como en otras que se están poniendo en marcha. Por último se presentan algunas ideas que pueden servir para la reflexión y la discusión. 
Nebot, C. P. (2018).

\section{Metodología}

Este trabajo utiliza como método el estudio de caso, habiéndose utilizado una combinación de técnicas de investigación social cuantitativas y cualitativas. La información cuantitativa recogida se empleó para elaborar las tablas y los cuadros presentes en éste artículo, mientras que la información cualitativa sirvió de base, por un lado, para reforzar los datos obtenidos, y por otro, para interpretar y contextualizar toda la información obtenida. El objeto del análisis es el primer Presupuesto Participativo Digital (PPD) puesto en marcha en el Ayuntamiento de Madrid. Los datos fueron recogidos mediante observación directa de la página web del PPD del Ayuntamiento de Madrid, en el período que va desde finales de febrero hasta mediados de agosto de 2016. Se realizó también un análisis documental de distintos documentos municipales (actas comisiones, actas plenos, reglamento de participación ciudadana...), políticos (programas presentados por los partidos políticos con representación municipal en las elecciones locales de 2015) y de noticias sobre el tema en la prensa escrita.

Además se programaron entrevistas en profundidad con el responsable del presupuesto participativo del equipo de gobierno, el Director General de Participación Ciudadana, y los concejales responsables de esa área de los otros tres partidos con representación municipal, el Partido Socialista Obrero Español (PSOE), el Partido Popular (PP) y Ciudadanos (C’s). Con disparidad en cuanto a los resultados de los contactos, en el caso del Director General y el concejal del PSOE hubo una gran disponibilidad y generosidad, sin embargo en el caso del Partido Popular, después de varios e-mail y llamadas, decidieron responder por escrito y con C's no se logró contactar al encontrarse la responsable del área de participación de baja maternal. Las entrevistas se celebraron entre finales de mayo y principios de junio, periodo de la fase de votación del presupuesto participativo. Para las entrevistas se elaboró un cuestionario y se grabaron en audio para poder analizar su contenido. Habría sido interesante poder entrevistar también a algunos de los ciudadanos participantes en el proceso, pero debido a la metodología utilizada no es posible conocer su identidad.

El análisis del éxito y continuidad de la experiencia puesta en marcha por el ayuntamiento de Madrid, se realizará a partir de tres hipótesis de trabajo: 
H.1 Voluntad política, considerando tanto los apoyos con los que cuenta para su aplicación como la categoría de "status político del PP" en la administración municipal;

H.2 Diseño institucional, en él no solo se evaluara el diseño que se ha utilizado sino también como se ha gestionado, elemento muy importante como veremos posteriormente;

H.3 Tecnología, al ser un proyecto que utiliza preferentemente las TIC es interesante valorar que herramientas incorpora y cuál es su uso.

\section{Análisis de la experiencia}

Antes de realizar el análisis del proyecto y con el fin de enmarcarlo en su contexto, presentamos algunas de las características de la ciudad de Madrid que pueden servir para entender mejor el funcionamiento de la experiencia de presupuesto participativo puesta en marcha en esa ciudad.

\subsection{Características de Madrid}

Madrid es la capital del Reino de España y la ciudad con más habitantes del país, 3.141.991. Durante veinticuatro años, desde 1991 a 2015, ha estado gobernada por el Partido Popular. Pero no fue hasta 2003, siendo Alcalde Alberto Ruiz-Gallardón, que se puso en marcha una política de participación ciudadana, que consistió en la creación de Consejos Territoriales en los veintiún distritos en que se divide administrativamente la ciudad (ver imagen 1), un Consejo Director, los Planes Especiales de Inversión y los Planes de Barrio. Estos últimos planes, los de Inversión y de Barrios, fueron el resultado de un acuerdo suscrito entre el Ayuntamiento de Madrid y la Federación Regional de Asociaciones de Vecinos de Madrid (FRAVM), siendo estos los interlocutores principales. Era, por tanto, un modelo de participación territorial y con actores colectivos. 
Nebot, C. P. (2018).

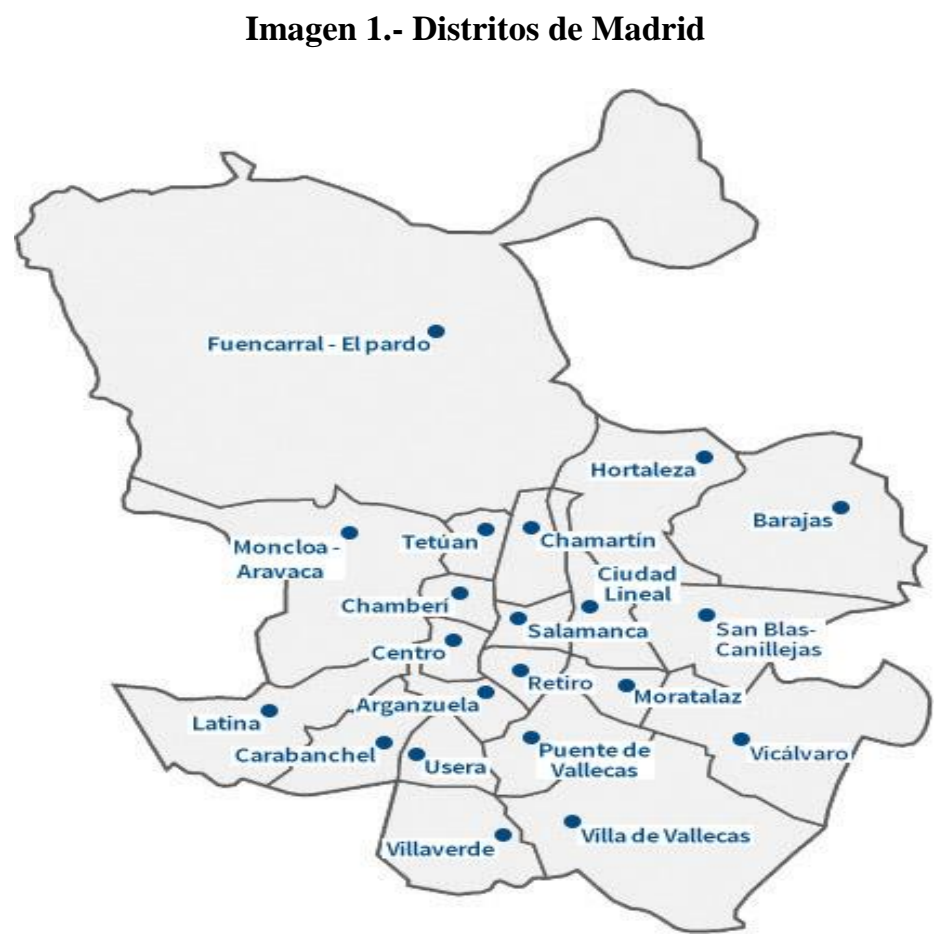

En las últimas elecciones municipales de 2015 con la entrada de los nuevos partidos políticos (Podemos y Ciudadanos) y la formación de una candidatura ciudadana, la situación política cambio. Como se puede apreciar en la tabla 1 el partido ganador fue, otra vez, el Partido Popular pero el pacto de investidura que firmaron Ahora Madrid, candidatura ciudadana resultado de la confluencia de Ganemos Madrid con Podemos, y el PSOE le dieron la Alcaldía a la candidata de Ahora Madrid, Manuela Carmena. Uno de los puntos del pacto que firmaron era la puesta en marcha del presupuesto participativo, proyecto que los dos llevaban en sus programas electorales.

Tabla 1.- Resultados Elecciones Municipales Madrid 2015

\begin{tabular}{|l|c|c|c|}
\hline Partido & Concejales & Votos & Porcentaje \% \\
\hline PP & 21 & 563.292 & 34.55 \\
\hline Ahora Madrid & 20 & 519.210 & 31.85 \\
\hline PSOE & 9 & 249.152 & 15.28 \\
\hline C's & 7 & 186.059 & 11.41 \\
\hline UPyD & 0 & 29.823 & 1.83 \\
\hline IU-Los Verdes & 0 & 27.869 & 1.71 \\
\hline
\end{tabular}

Fuente: Ministerio del Interior.

El nuevo gobierno municipal, formado exclusivamente por Ahora Madrid, organizó el ayuntamiento en nueve áreas separando la participación ciudadana en dos áreas: el Área de Coordinación Territorial y Asociaciones que se encarga de la participación colectiva y el Área 
de Participación Ciudadana, Transparencia y Gobierno Abierto que lo hace de la participación individual. Habiendo pasado todo el personal de participación ciudadana que había con el anterior gobierno municipal al área de coordinación territorial (Concejal Pablo Soto en CPCTGA $\mathrm{n}^{\mathrm{o}}$ 1175), por lo que el área de participación individual no contaba con personal especializado en el tema.

Poco después de tomar posesión el nuevo gobierno, en mayo de 2015, los concejalespresidentes de tres distritos de la ciudad, Arganzuela, Tetuán y Usera, crearon unos grupos motores (de los que no existe información) para poner en marcha experiencias piloto de presupuesto participativo, proyectos que, como nos manifestaron los entrevistados, fueron concebidos autónomamente en cada distrito por el concejal responsable.

Estas experiencias tenían varias características comunes y alguna diferencia. En las tres se crearon Grupos Motores, la cantidad destinada era de $100.000 €$ por distrito, el período de funcionamiento muy corto (de octubre a diciembre), cada persona podía presentar tres propuestas, la presentación era presencial u online, las propuestas debían ser de competencia del distrito, se realizaban informes técnicos de las propuestas y podían votar las personas que residieran o trabajaran en el distrito. En lo que no existió concordancia es en la edad mínima para participar, que va desde los 12 años en Usera, a los 14 en Arganzuela o los 16 en Tetuán y en el número de propuestas a priorizar: 3 en Tetuán y 5 en Arganzuela y Usera. La participación fue pequeña, en ninguno de los distritos se llegó al $0.5 \%$ de la población. Estos proyectos no se conocían por la Dirección General de Participación Ciudadana, que creían que los había llevado a cabo el anterior equipo de gobierno del Partido Popular.

El 22 de febrero de 2016 la Junta de Gobierno de la Ciudad de Madrid aprueba, basándose en el art. 27 del Reglamento Orgánico de Participación Ciudadana, las directrices para el desarrollo de los presupuestos participativos. La utilización de esa figura jurídica, la audiencia pública, no parece la más adecuada para el presupuesto participativo, como nos manifestó el responsable del PSOE, pero desde el equipo de gobierno la ven como la única que se ajusta al proyecto. A partir de ese momento se inicia, a través de la web de gobierno abierto, el procedimiento de audiencia pública sobre el presupuesto participativo correspondiente al ejercicio 2017. 
Nebot, C. P. (2018).

\subsection{Análisis de las variables}

Ahora pasaremos a analizar cada una de las variables estudiadas en nuestra investigación.

\subsubsection{Voluntad Política}

Ahora Madrid ha contado desde el principio del proceso con el apoyo del Partido Socialista Obrero Español (PSOE). Este apoyo se ha visualizado no solo en los votos sino también en las ruedas de prensa en las que se ha hablado del presupuesto participativo, donde ha estado siempre la portavoz del PSOE, Purificación Causapié, al lado de la alcaldesa, Manuela Carmena.

El respaldo del PSOE al proyecto, según su portavoz, se debe a dos razones: porque así constaba en uno de los puntos del acuerdo de investidura y porque los socialistas "siempre vamos a estar en cualquier consenso que tenga como objeto la profundización de la salud democrática de la ciudad o que reduzca las desigualdades y combata las injusticias que aún persisten en la ciudad". Habiendo valorado positivamente, desde el principio, la puesta en marcha del proyecto e involucrándose en él.

Los socialistas tienen además mucha experiencia, ya que ayuntamientos gobernados por ellos, en solitario o en coalición con Izquierda Unida, fueron pioneros en implantar los presupuestos participativos en España. De la mano de gobiernos socialistas, ciudades tan importantes como Sevilla, Albacete, San Sebastián, Getafe, Leganés, Sabadell, Leganés, Segovia, Torrellano, Elche, Algeciras o Novelda entre otras, asumieron un proyecto que no sólo supone otra manera de gastar, sino que implica otra manera de gobernar. Y, en el ámbito internacional, son muchos los ejemplos de ciudades regidas por socialistas y que cuentan con presupuesto participativo, Lisboa, Roma, y París son algunos ejemplos de ello.

Ello no les impide plantear críticas a la gestión del proceso, que consideran manifiestamente mejorable tanto en la parte online como la parte presencial, es decir, los encuentros y reuniones en los distritos. Por ello se han comprometido, y así lo han trasladado en numerosas ocasiones a las asociaciones y a los ciudadanos, a recoger sugerencias y propuestas de mejora para apoyar este proceso participativo en años sucesivos. Otra de sus críticas es sobre el tipo de publicidad usada para fomentar la democracia participativa, 
publicidad que piensan ha podido inducir a engaño o confusión y además ha generado crispación.

La posición de Ciudadanos (C's) sin ser tan activa como la del PSOE, ya que no han firmado ningún pacto, es también positiva. Ciudadanos defiende que haya participación, pero que ésta "sea informada, explicando y haciendo pedagogía sobre dónde llegan las competencias del Ayuntamiento" porque, de lo contrario, piensan que se caería en la "frustración". Para ellos la democracia participativa no debe sustituir a la representativa sino que debe complementarla.

El Partido Popular es el que plantea una mayor oposición al proyecto, pero no sólo a él sino que lo suele hacer a toda medida tomada por el gobierno de la ciudad, siendo su portavoz, Esperanza Aguirre, la que adopta posiciones más radicales. El Partido Popular tildó desde el principio de "falso referéndum" los presupuestos participativos de la ciudad de Madrid, manifestando que con ellos se pretendía "legitimar con una falsa participación ciudadana las decisiones que el propio equipo de Gobierno ya tiene tomadas". Señalando también que pese a que están a favor "de la participación ciudadana", están en contra de que "se sustituya la democracia representativa por una falsa democracia participativa" у considerando que al no marcar un mínimo de ciudadanos que tienen que participar, el presupuesto participativo era una "tomadura de pelo". Esperanza Aguirre indicó además que le llamaba poderosamente la atención que, dado el carácter 'participativo' de esta iniciativa, se haya excluido de la misma al PP y a C's, ya que se supone que esta medida de 'Gobierno Abierto' debe incluir a todos los grupos que representan a los madrileños.

Aunque sería deseable para la sostenibilidad del proceso que estuvieran implicados todos los grupos políticos del ayuntamiento, la experiencia cuenta con un apoyo mayoritario y además organizativamente se encuentra ubicado en un área importante.

\subsubsection{Diseño Institucional}

El modelo que instauran es según Pablo Soto, Concejal de Participación Ciudadana, Transparencia y Gobierno Abierto, "el que está ahora mismo extendiéndose por toda Europa y más allá, bueno, es el modelo de presupuestos participativos que está llevando a cabo París por ejemplo, Reikiavik, Grenoble o Nueva York, en el cual, pues, cualquier persona o colectivo hace una propuesta de nueva inversión" (CPCTGA nº 1175). 
Nebot, C. P. (2018).

El modelo tiene las siguientes características: a) todas las personas empadronadas en Madrid y mayores de 16 años pueden presentar propuestas; b) las propuestas se pueden presentar a través de la web https://decide.madrid.es/ o en cualquiera de las 26 oficinas de Atención a la Ciudadanía; c) el proceso se divide en cuatro fases: recogida de propuestas, apoyos, evaluación y votación final; d) se puede votar en la red o presencialmente; e) para votar en la web es necesario registrarse, posteriormente se verifica y se facilita un código seguro que se comunica a través del teléfono móvil o el correo; para el voto presencial se pide el DNI; f) las propuestas deben ser de inversión; g) se destinan 60 millones de euros del capítulo de inversiones, de ellos un $40 \%$ es para proyectos que afecten a toda la ciudad y el $60 \%$ restante a propuestas localizadas en los distritos; h) la distribución entre los distritos se hace de forma directamente proporcional a la población de cada uno e inversamente proporcional a la renta per cápita de cada distrito; i) las propuestas deben presentarse individualmente aunque puede figurar que proviene de una asociación o colectivo; j) las Juntas de Distrito podrán en marcha espacios presenciales para desarrollar todo el proceso; k) se votan propuestas de ciudad y de distritos, pero solo se puede elegir un distrito; 1) se puede votar por delegación.

La cantidad destinada al proyecto por el gobierno municipal era de 100 millones pero en la negociación con el PSOE y a instancias de ellos, decidieron reducirla a 60 millones. La razón esgrimida por el PSOE para que fuera una cantidad menor es que era un proyecto que había que testar y que siempre habría tiempo para ir subiendo la cantidad. Esa cantidad supone un $20 \%$ del capítulo destinado a inversiones y un $1,34 \%$ del total del gasto del presupuesto municipal de 2016. Capítulo, el de inversiones, que ha aumentado un 2,3\% de 2015 a 2016 como se puede en la tabla 2.

Tabla 2.- Gastos Presupuestados Ayuntamiento de Madrid 2013-2016

\begin{tabular}{|l|r|r|r|r|}
\hline \multicolumn{1}{|c}{ CAPITULO } & \multicolumn{1}{c}{$\mathbf{2 0 1 3}$} & $\mathbf{2 0 1 4}$ & $\mathbf{2 0 1 5}$ & $\mathbf{2 0 1 6}$ \\
\hline 1. Personal & 1.109 .352 .117 & 1.100 .500 .007 & 1.130 .922 .240 & 1.159 .444 .271 \\
\hline 2. Bienes Corrientes y Servicios & 1.491 .370 .639 & 1.542 .410 .719 & 1.525 .474 .106 & 1.617 .817 .074 \\
\hline 3. Financieros & 301.766 .718 & 312.427 .953 & 206.525 .588 & 158.811 .384 \\
\hline 4. Transferencias corrientes & 496.046 .274 & 480.277 .863 & 519.182 .539 & 596.977 .422 \\
\hline 5. Fondos Cont. e imprevistos & 0 & 0 & 19.425 .681 & 17.082 .728 \\
\hline OPERACIONES CORRIENTES & 3.398 .535 .748 & 3.435 .616 .542 & 3.401 .530 .154 & 3.550 .132 .879 \\
\hline 6. Inversiones reales & 117.334 .309 & 170.660 .013 & 206.538 .407 & 303.243 .071 \\
\hline 7. Transferencias de Capital & 46.158 .880 & 32.390 .674 & 39.898 .815 & 69.094 .500 \\
\hline
\end{tabular}




\begin{tabular}{|l|r|r|r|r|}
\hline OPERACIONES DE CAPITAL & 163.493 .189 & 203.050 .687 & 246.437 .222 & 372.337 .571 \\
\hline $\begin{array}{l}\text { 8. Activos Financieros } \\
\text { 9. Pasivos Financieros }\end{array}$ & 0 & 0 & 0 & 3.060 .000 \\
\hline $\begin{array}{l}\text { OPERACIONES } \\
\text { FINANCIERAS }\end{array}$ & 732.974 .309 & 803.646 .526 & 734.617 .652 & 557.970 .015 \\
\hline TOTAL & 4.295 .003 .246 & 4.442 .313 .755 & 4.382 .585 .028 & 4.483 .500 .465 \\
\hline
\end{tabular}

Fuente: Ayuntamiento de Madrid.

El proyecto tiene un carácter redistributivo, asignando más dinero a los distritos con más población y menos renta per cápita, en la tabla 3 podemos ver las cantidades asignadas a los distritos en el presupuesto participativo y algunos datos que nos permiten conocer mejor las características de estos. Hay cuatro distritos señalados en negrita (Carabanchel, Puente de Vallecas, Usera y Villaverde) que son los que tienen una renta per cápita más baja, el paro más alto y un número importante de AA.VV. Por otro lado, hay cinco distritos señalados en cursiva (Barajas, Chamartín, Chamberí, Retiro y Salamanca) con una renta per cápita más alta, menos paro y menos AA.VV.

Tabla 3.- Datos de los distritos de Madrid

\begin{tabular}{|c|c|c|c|c|c|c|}
\hline Distritos & $\begin{array}{c}\text { Cantidad } \\
\text { asignada PP }\end{array}$ & $\begin{array}{l}\text { Población } \\
2016\end{array}$ & Asociaciones & $\begin{array}{c}\text { AA.V } \\
\text { V }\end{array}$ & $\begin{array}{c}\text { Paro/ } \\
\text { marzo } \\
2016\end{array}$ & $\begin{array}{c}\text { Renta per } \\
\text { cápita } 2011\end{array}$ \\
\hline Arganzuela & 1.556 .169 & 150.177 & 74 & 5 & 8.925 & 23.356 \\
\hline Barajas & 433.589 & 45.747 & 37 & 7 & 2.351 & 25.297 \\
\hline Carabanchel & 3.247 .830 & 240.230 & 110 & 13 & 20.185 & 18.049 \\
\hline Centro & 1.353 .966 & 131.106 & 199 & 9 & 9.613 & 23.084 \\
\hline Chamartín & 1.313 .747 & 141.435 & 64 & 8 & 5.768 & 25.815 \\
\hline Chamberí & 1.259 .587 & 137.286 & 84 & 4 & 6.153 & 25.984 \\
\hline Ciudad Lineal & 2.287 .757 & 211.736 & 105 & 5 & 13.771 & 22.182 \\
\hline $\begin{array}{l}\text { Fuencarral-El } \\
\text { Pardo }\end{array}$ & 2.441 .608 & 232.889 & 123 & 16 & 11.249 & 23.371 \\
\hline Hortaleza & 1.827 .228 & 174.343 & 80 & 11 & 9.680 & 23.229 \\
\hline Latina & 2.927 .200 & 234.222 & 119 & 18 & 17.067 & 19.333 \\
\hline $\begin{array}{l}\text { Moncloa- } \\
\text { Aravaca }\end{array}$ & 1.129 .851 & 115.901 & 77 & 9 & 5.132 & 23.941 \\
\hline Moratalaz & 1.067 .341 & 94.813 & 52 & 1 & 6.365 & 21.231 \\
\hline Puente de & 3.349 .186 & 226.135 & 124 & 15 & 22.742 & 16.730 \\
\hline
\end{tabular}

R.G. Secr., GESEC, São Paulo, v. 9, n. 1, p 265-292, jan./abr. 2018. 
Nebot, C. P. (2018).

\begin{tabular}{|l|r|r|r|r|r|r|}
\hline Vallecas & & & & & \\
Retiro & 1.075 .155 & 117.934 & 64 & 4 & 5.133 & 26.409 \\
\hline Salamanca & 1.286 .657 & 142.388 & 74 & 2 & 6.116 & 26.265 \\
\hline San Blas- & 1.712 .043 & 152.447 & 82 & 12 & 11.195 & 21.556 \\
Canillejas & & & & & & \\
Tetuán & 1.677 .256 & 150.860 & 83 & 7 & 10.449 & 22.025 \\
\hline Usera & 1.923 .216 & 132.744 & 93 & 11 & 11.863 & $\mathbf{1 6 . 6 9 1}$ \\
Vicálvaro & 879.529 & 69.363 & 32 & 4 & 5.391 & 19.537 \\
Villa de & 1.220 .810 & 99.636 & 59 & 4 & 8.374 & 20.402 \\
Vallecas & & & & & & \\
Villaverde & 2.030 .275 & 140.599 & 13 & 13 & 13.524 & $\mathbf{1 6 . 9 7 0}$ \\
\hline TOTAL & 36.000 .000 & 3.141 .991 & 1.819 & 178 & 211.046 & \\
\hline
\end{tabular}

Fuente: Elaboración propia.

Ese carácter redistributivo no se produce, sin embargo, en el caso de las inversiones territorializadas, es decir por distritos, que realiza el ayuntamiento de Madrid, inversiones que en el presupuesto de 2016 suponen el 47\% del capítulo de inversiones. En la tabla 4 podemos ver las inversiones por habitante y distrito territorializadas y del PP. En ella se puede ver que los distritos con renta per cápita más baja y más paro (en negrita) son los que tienen una mayor inversión por habitante en el PP, pero en cambio no en la inversión territorializada, excepto en el caso de Usera.

Tabla 4.- Inversión por habitante y distrito territorializada y en el PP.

\begin{tabular}{|l|r|r|}
\hline \multicolumn{1}{|c|}{ Distritos } & $\begin{array}{c}\text { Inversión por habitante y } \\
\text { distrito (en euros) en el }\end{array}$ & $\begin{array}{c}\text { Inversión por habitante } \\
\text { Presupuesto Participativo (en } \\
\text { Presupuesto de 2016 }\end{array}$ \\
\hline $\begin{array}{l}\text { Arganzuela } \\
\text { Barajas }\end{array}$ & 104,10 & 10,36 \\
\hline Carabanchel & 125,72 & 9,48 \\
\hline Centro & 17,62 & $\mathbf{1 3 , 5 2}$ \\
\hline Chamartín & 5,67 & 10,33 \\
\hline Chamberí & 8,90 & 9,29 \\
\hline Ciudad Lineal & 14,95 & 9,17 \\
\hline Fuencarral-El Pardo & 14,74 & 10,80 \\
\hline Hortaleza & 16,78 & 10,48 \\
\hline Latina & 131,73 & 10,48 \\
\hline Moncloa-Aravaca & 28,24 & 12,50 \\
\hline
\end{tabular}

R.G. Secr., GESEC, São Paulo, v. 9, n. 1, p 265-292, jan./abr. 2018. 


\begin{tabular}{|l|r|r|}
\hline Moratalaz & 58,18 & 11,26 \\
\hline Puente de Vallecas & 70,03 & $\mathbf{1 4 , 8 1}$ \\
\hline Retiro & 22,48 & 9,12 \\
\hline Salamanca & 13,42 & 9,04 \\
\hline San Blas-Canillejas & 26,71 & 11,23 \\
\hline Tetuán & 70,23 & 11,12 \\
\hline Usera & 113,09 & $\mathbf{1 4 , 4 9}$ \\
Vicálvaro & 72,18 & 12,68 \\
\hline Villa de Vallecas & 61,49 & 12,25 \\
\hline Villaverde & 53,58 & $\mathbf{1 4 , 4 4}$ \\
\hline
\end{tabular}

Fuente: Elaboración propia.

Antes de que comience la primera fase de recogida de propuestas, el 22 de febrero, se realizó una campaña de comunicación con carteles en los autobuses municipales, en las paradas de autobuses y en las pantallas fijas que hay por la ciudad. Campaña que no consiguió llegar a la población, por lo que la ampliaron en la fase de votación con un autobús que iba recorriendo la ciudad y que además de informar sobre el proyecto ayudaba a los vecinos que tenían problemas para votar online. En opinión del Director General el tamaño de la ciudad y el tener los medios de comunicación en contra ha dificultado mucho su difusión, en cambio para otros entrevistados la comunicación no estuvo bien organizada.

En la fase de presentación de propuestas participaron 20.000 personas y se presentaron, según fuentes del gobierno municipal, 5.000 propuestas (que se convirtieron en 5.184 cuando terminó todo el proceso y facilitaron los datos finales), que fueron clasificadas por los ocho funcionarios de la Dirección General de Participación Ciudadana. Funcionarios que, como señalo el Director General, se ha mostrado que son escasos para el proyecto y que además no tenían ningún conocimiento sobre el tema. De ellas, 1.376 propuestas no pasaron a la siguiente fase al no cumplir los requisitos exigidos (ser legales, concretas, competencia del ayuntamiento y no superar el tope del presupuesto), es decir solo pasaron 3.624 propuestas. A partir de ese momento fue cuando nos encontramos con problemas: en primer lugar porque existía un desfase de 301 propuestas entre las que indicaba el gobierno municipal y las que hemos encontrado en la página web; en segundo lugar porque no son capaces de explicar esa diferencia y; en tercer lugar porque no explican las razones para la eliminación de algunas propuestas. 
Nebot, C. P. (2018).

Durante esta fase de propuestas las Juntas de Distrito convocaron a los vecinos y a los colectivos a unos espacios presenciales para debatir y construir colectivamente. El número de reuniones de estos espacios fue ampliándose, desde una en algunos casos hasta cuatro en otros. Pero en ningún momento se ha conocido cuantas personas participaron, ni si eran colectivos o ciudadanos individuales, ni los resultados alcanzados. Alguna de las personas entrevistadas nos dijo que esas reuniones se convocaron, por el área de Coordinación Territorial y Asociaciones, para presentar los planes de distrito, que son propuestas del gobierno municipal que se explican a los vecinos, y que se mezcló con el presupuesto participativo, lo que creo mucha confusión y rechazo. Lo que no logramos conocer es si se hizo con la intención de mezclarlo, por falta de tiempo o por desconocimiento de la dinámica. Lo cierto es que parece que fue poca gente y que además los vecinos se enfadaron por que hubieran mezclado los dos proyectos. Según el concejal responsable del área, Pablo Soto, ha habido 81 reuniones plenarias con 2.812 participantes contados, es decir una media de 35 personas por reunión. Aunque señaló también que "es dificil saber cuántos han sido porque esos espacios se ha pretendido, y así ha ocurrido en la mayoría, que sean autogestionados, lo que significa que ellos mismos se convocan para siguientes reuniones, ellos mismos se organizan en grupos de trabajo y en esas reuniones no asistimos nosotros como personal municipal"(CPCTGA nº 1303) .

La segunda fase de apoyos comenzó el 1 de abril y duró quince días. En ella cada persona podía apoyar diez propuestas para toda la ciudad y diez en un distrito concreto, pero solo en uno. Al finalizar esta segunda etapa el gobierno facilitó los siguientes datos: habían participado 23.825 personas y se habían registrado 168.811 apoyos a las propuestas. Al colgar, al final del proceso, los datos totales en la web el número de personas que habían apoyado las propuestas era de 18.709. Como en la anterior fase existe un desfase de datos, a pesar de que ambos provienen de la misma fuente, el gobierno municipal. Hortaleza y Latina fueron los distritos donde hubo un número mayor de participantes (el 15\% y el 10\% del total, respectivamente).

Al cerrar esta fase hasta las propuestas más valoradas habían conseguido un número de apoyos bajo, como se puede ver en la tabla 5. Pero lo más curioso es que ya cerrado el plazo cambiaron las propuestas más apoyadas y hasta la cantidad de apoyos que tenían algunas propuestas, normalmente aumentando el número de apoyos excepto en el caso de Hortaleza que cambió una propuesta con 1.032 apoyos por una con 450. 
Tabla 5.- Apoyos de las propuestas mejor valoradas

\begin{tabular}{|c|c|c|}
\hline DISTRITOS & $\begin{array}{l}\text { Apoyos propuestas } \\
\text { mejor valoradas }\end{array}$ & $\begin{array}{l}\text { Apoyos propuestas mejor } \\
\text { valoradas cerrada la fase }\end{array}$ \\
\hline Arganzuela & 226 & 264 \\
\hline Barajas & 56 & 112 \\
\hline Carabanchel & 161 & 819 \\
\hline Centro & 310 & 348 \\
\hline Chamartín & 142 & 142 \\
\hline Chamberí & 166 & 160 \\
\hline Ciudad Lineal & 235 & 244 \\
\hline Fuencarral-El Pardo & 183 & 282 \\
\hline Hortaleza & 1032 & 450 \\
\hline Latina & 496 & 549 \\
\hline Moncloa-Aravaca & 100 & 132 \\
\hline Moratalaz & 201 & 216 \\
\hline Puente de Vallecas & 242 & 242 \\
\hline Retiro & 155 & 188 \\
\hline Salamanca & 125 & 134 \\
\hline San Blas-Canillejas & 178 & 214 \\
\hline Tetuán & 136 & 169 \\
\hline Usera & 108 & 148 \\
\hline Vicálvaro & 119 & 119 \\
\hline Villa de Vallecas & 215 & 281 \\
\hline Villaverde & 170 & 135 \\
\hline Ciudad & 2349 & 2950 \\
\hline
\end{tabular}

Fuente: Elaboración propia.

La explicación que se nos dio es que se habrían incorporado datos, que habían llegado tarde, de los apoyos realizados presencialmente en las Oficinas de Atención a la Ciudadanía. Pero no hubo en la web ninguna indicación al respecto.

En ese momento en el apartado delegación, figura que no nos han sabido explicar y que resulta realmente extraña, había propuestas en dieciocho distritos y para toda la ciudad.

En la tercera fase que duró casi un mes (del 16 de abril hasta el 14 de mayo) un equipo técnico formado por funcionarios de las áreas y de los distritos (207 según la información del gobierno municipal), coordinados por el Área de Participación Ciudadana, evaluaron las propuestas para confirmar que estas eran válidas, viables y legales. Las propuestas que eran 
Nebot, C. P. (2018).

similares entre sí eran notificadas a sus autores ofreciéndoles la posibilidad de unificarlas. Al finalizar este periodo se debían publicar todas las propuestas, tanto las aprobadas como las rechazadas, con sus informes y valoraciones correspondientes. Algo que no se cumplió del todo, ya que como informaron en la página web "pasaron a la votación final tantas propuestas viables cómo fue posible evaluar en el tiempo disponible, realizándose los estudios en el orden de apoyos decidido por la gente en la fase de preselección, intentando que hubiera un número de propuestas viable similar en cada distrito. Teniendo en cuenta que esto implica un número diferente de apoyos, estos han sido los valores de corte de número de apoyo para cada ámbito”.

Tabla 6.- Valores de corte

\begin{tabular}{|c|c|}
\hline ÁMBITO & APOYOS DEL CORTE \\
\hline Toda la ciudad & 52 \\
\hline Arganzuela & 40 \\
\hline Barajas & 10 \\
\hline Carabanchel & 37 \\
\hline Centro & 49 \\
\hline Chamartín & 31 \\
\hline Chamberí & 28 \\
\hline Ciudad Lineal & 43 \\
\hline Fuencarral-El Pardo & 56 \\
\hline Hortaleza & 39 \\
\hline Latina & 49 \\
\hline Moncloa-Aravaca & 57 \\
\hline Moratalaz & 16 \\
\hline Puente de Vallecas & 39 \\
\hline Retiro & 34 \\
\hline Salamanca & 26 \\
\hline San Blas-Canillejas & 24 \\
\hline Tetuán & 31 \\
\hline Usera & 34 \\
\hline Vicálvaro & 20 \\
\hline Villa de Vallecas & 24 \\
\hline Villaverde & 27 \\
\hline
\end{tabular}

Fuente: Ayuntamiento de Madrid.

Durante el estudio de las propuestas se reclasificaron las propuestas que correspondían a otro ámbito (por ejemplo una propuesta etiquetada en un distrito que correspondía realmente 
a otra zona, o una de un distrito que implicaba a toda la ciudad y no sólo a ese distrito). Además las propuestas de distrito cuyo coste era superior a la cantidad de dinero asignada a ese distrito la clasificaron como propuesta relativa a toda la ciudad. Eso se hacía para no eliminarlas de la fase de votación por exceso de coste, pero trasgredía las propias normas que se habían dado, ya que en ellas se señalaba que las propuestas para la ciudad no podían estar localizadas en un distrito especifico. De esa forma todas las propuestas de bibliotecas o polideportivos de los distritos pasaron a ser propuestas de ciudad.

Cuando se publicaron las propuestas se vio que algunas habían desaparecido, aunque no se encontraron entre las no viables. Tampoco se encontraron los informes y valoraciones de todas las propuestas del período de apoyos, suponemos que también sería por falta de tiempo o de personal. Al final solo se evaluaron 970 propuestas. De ellas solo llegaron a la votación final, según los datos recogidos en la web, 750 propuestas, el 19\% del total de propuestas apoyadas. Para toda la ciudad 170 propuestas y las restantes 580 en los distritos. Aunque los datos facilitados por el gobierno municipal hablan de 623 propuestas.

La votación final duró un mes y medio, del 15 de mayo hasta el 30 de junio, y según datos del gobierno municipal votaron 28.819 personas y hubo 206 propuestas ganadoras, de ellas 41 propuestas de asociaciones y 66 de los espacios presenciales. Según los datos recogidos en la web hay 215 propuestas ganadoras, cuatro de ellas con coste cero. Al estar relacionadas con el coste de las propuestas nos encontramos con distritos con una propuesta (Hortaleza) y otra con veintidós (Chamartín).

También hay que señalar que se podía votar de dos formas: eligiendo personalmente una a una las propuestas o votando en bloque las propuestas que se encontraban en el apartado de delegación. Apartado del que no se tenía ninguna información en la web sobre su procedencia y que tampoco conseguimos conocer durante nuestra investigación.

Durante el tiempo de la votación en el espacio delegación solo estaban las propuestas de ciudad y las de nueve de los veintiún distritos de la ciudad, varios días después de que se conocieran los resultados de la votación había en ese espacio propuestas de ocho distritos más. Solo cuatro distritos no tenían propuestas en el apartado delegación (Hortaleza, Moncloa, Moratalaz y Tetuán), aunque puede que más tarde las tuvieran.

A pesar de eso, muchas de las propuestas aprobadas, como podemos ver en la tabla 7 , corresponden a las propuestas del apartado delegación (el 60\%), sobre todo en el caso de las propuestas de ciudad. De las 22 propuestas aprobadas en el ámbito de ciudad, 19 provenían del espacio delegación, lo que supone el $86 \%$ del total. Según el concejal responsable del área 
Nebot, C. P. (2018).

de los 206 proyectos ganadores " 41 de ellos fueron propuestos por asociaciones a título no presencial, no en los espacios presenciales pero por asociaciones, y 66 proyectos fueron presentados en los espacios presenciales, fueron promovidos por los espacios de debate presenciales. En total, 107 proyectos de estos 206 vienen de estos procesos colectivos, lo que pone un poco en perspectiva que la participación como un proceso deliberativo ha funcionado en tanto en cuanto la mayoría de los proyectos ganadores nacen de procesos colectivos" (CPCTGA n $\left.{ }^{\circ} 1315\right)$.

Tabla 7.- Propuestas aprobadas

\begin{tabular}{|c|c|c|}
\hline Distritos & $\begin{array}{l}\text { Número de propuestas } \\
\text { aprobadas }\end{array}$ & $\begin{array}{c}\text { Número de propuestas incluidas en } \\
\text { delegación }\end{array}$ \\
\hline Arganzuela & 6 & 4 \\
\hline Barajas & 8 & 6 \\
\hline Carabanchel & 10 & 9 \\
\hline Centro & 10 & 4 \\
\hline Chamartín & 12 & error \\
\hline Chamberí & 7 & 4 \\
\hline Ciudad Lineal & 10 & 7 \\
\hline Fuencarral-El Pardo & 15 & 10 \\
\hline Hortaleza & 1 & No hay \\
\hline Latina & 4 & 1 \\
\hline Moncloa-Aravaca & 11 & No hay \\
\hline Moratalaz & 9 & No hay \\
\hline Puente de Vallecas & 7 & 2 \\
\hline Retiro & 7 & 5 \\
\hline Salamanca & 7 & 5 \\
\hline San Blas-Canillejas & 11 & 9 \\
\hline Tetuán & 8 & No hay \\
\hline Usera & 7 & 5 \\
\hline Vicálvaro & 5 & 4 \\
\hline Villa de Vallecas & 8 & 0 \\
\hline Villaverde & 11 & 6 \\
\hline Ciudad & 22 & 19 \\
\hline Total & 167 & 100 \\
\hline
\end{tabular}

Fuente: Elaboración propia

R.G. Secr., GESEC, São Paulo, v. 9, n. 1, p 265-292, jan./abr. 2018. 
En el período de votación tampoco se podía conocer que apoyos habían tenido cada propuesta pues se retiraron de la web, cuando preguntamos en la entrevista al Director General nos dijo que fue un tema que discutieron en las reuniones, pero que decidieron hacerlo de esta forma por miedo a los lobbys.

El total de participantes en el proceso fue de 45.522 personas, un éxito para el responsable del área al ser un número mayor que el que consiguieron en su primer año (2014) en la experiencia de Paris, que fue de 40.745. Algo que es cierto pero que tiene un pequeño truco, las dos ciudades no tienen el mismo número de habitantes. En Paris, con una población de 2.240.621 habitantes, la participación suponía un 1,82\% de su población, en cambio, en Madrid con 3.141.991 habitantes, supone el 1,45\%.

De los 60 millones de euros destinados al proyecto han sobrado $1.293 .330 €$, cantidad que no conocemos a que se dedicará. En el distrito que menos ha sobrado es en Villaverde (275 euros), en cambio en la Latina, San Blas-Canillejas, Vicalvaro y Villa de Vallecas ha sido más de 100.000 euros en cada uno de ellos.

Estas propuestas aprobadas pasaran directamente al presupuesto municipal y esperamos que se publique en el espacio del presupuesto participativo de la web, a partir de ahí se hará un seguimiento de cada una de las propuestas donde se irá detallando su recorrido.

El siguiente paso era realizar durante los meses de septiembre y octubre una evaluación de todo el proceso, que esperamos que sea pública. Serán, en palabras del Concejal Pablo Soto, "una serie de reuniones y debates al respecto de cómo ha ido este proceso, no tanto desde el punto de vista de la institución sino desde el punto de vista de la propia ciudadanía, y que esa última fase pueda desembocar en algunas recomendaciones que se puedan tener en cuenta para mejorar el proceso de cara al año siguiente" (CPCTGA n ${ }^{\circ}$ 1315). Está bien que los ciudadanos evalúen el proceso pero creemos que la institución debe hacer también una evaluación de su gestión del proceso.

Del análisis de la metodología se puede extraer que está no ha sido la más acertada quizá por falta de tiempo y de personal especializado. Además la gestión que el ayuntamiento ha hecho ha sido muy confusa, creemos que por varias razones: desconocimiento del tema, falta de personal, mucha prisa por ponerlo en marcha, falta de experiencia de gestión, etc. Suponemos y esperamos que muchos de los errores los irán corrigiendo con el tiempo. 
Nebot, C. P. (2018).

\subsubsection{Tecnología}

Desde la mitad de los años 90, los modelos de participación comenzaron a encontrar en las tecnologías, cada vez más avanzadas, la solución a alguno de sus problemas (accesibilidad, información, transparencia, control...). Una de estas experiencias de participación ciudadana, quizá la más conocida y de mayor éxito, el Presupuesto Participativo, no ha permanecido ajena a las tecnologías. En 2001, dos ciudades brasileñas, Ipatinga y Porto Alegre, está última la referencia mundial del Presupuesto Participativo comenzaron a realizar pequeñas ensayos con las tecnologías. En ambas, este primero paso supuso, básicamente, la posibilidad de que los ciudadanos enviaran online propuestas al PP presencial, con el fin de reforzarlo. Pero no fue hasta 2006, con la puesta en marcha del PP digital de Belo Horizonte, que la posibilidad de usar las tecnologías en los procesos de presupuesto participativo comenzó a coger fuerza. Habiendo, en estos momentos, más de 200 experiencias de presupuesto participativo en todo el mundo que utilizan total o parcialmente la tecnología.

El caso del PP de Madrid es un modelo mixto, en que se utilizan tanto medios online como presenciales, aunque mayoritariamente online (Sampaio, 2014). La web de Gobierno Abierto del Ayuntamiento de Madrid, responsabilidad del concejal del área, incorpora en ella a http://decide.madrid.es. En Decide Madrid hay varios apartados, como se puede ver en la Figura 2, dedicados a distintos temas: debates, propuestas, procesos sectoriales y presupuestos participativos.

Nada más entrar en el apartado presupuestos participativos nos encontrábamos con un diseño muy simple, con colores muy neutros en los que prima el azul. En él se pueden ver las distintas fases del proceso, que van cambiando de color, de blanco a amarillo, según la fase en la que nos encontramos. Luego pasamos a la información donde se explica que es el presupuesto participativo, en que consiste el proyecto y cuál es su metodología. En esta parte, en otras experiencias, suele haber además un video que explica todo el proceso y que permite llegar mejor a un número mayor de personas.

La información sobre el presupuesto participativo y las experiencias que consideran de referencia es muy escasa, podían haberla ampliado o poner enlaces para que los ciudadanos las pudieran conocer. La principal referencia del concejal del área, la plataforma Retri Reykjavík (https://betrireykjavik.is/), fue creada en 2010 para recibir colaboraciones de los ciudadanos para cambiar y mejorar la ciudad, con una población de 118.500 personas, y terminó siendo utilizada para el presupuesto participativo. La mayor innovación de esta 
plataforma es el sistema de debates que no parece que se haya utilizado en el caso de Madrid. No existen referencias en la web de Madrid de experiencias brasileñas, cuna del presupuesto participativo, ni de experiencias que se hayan realizado en España anteriormente.

Figura 2: Página de inicio del Presupuesto Participativo del Ayuntamiento de Madrid

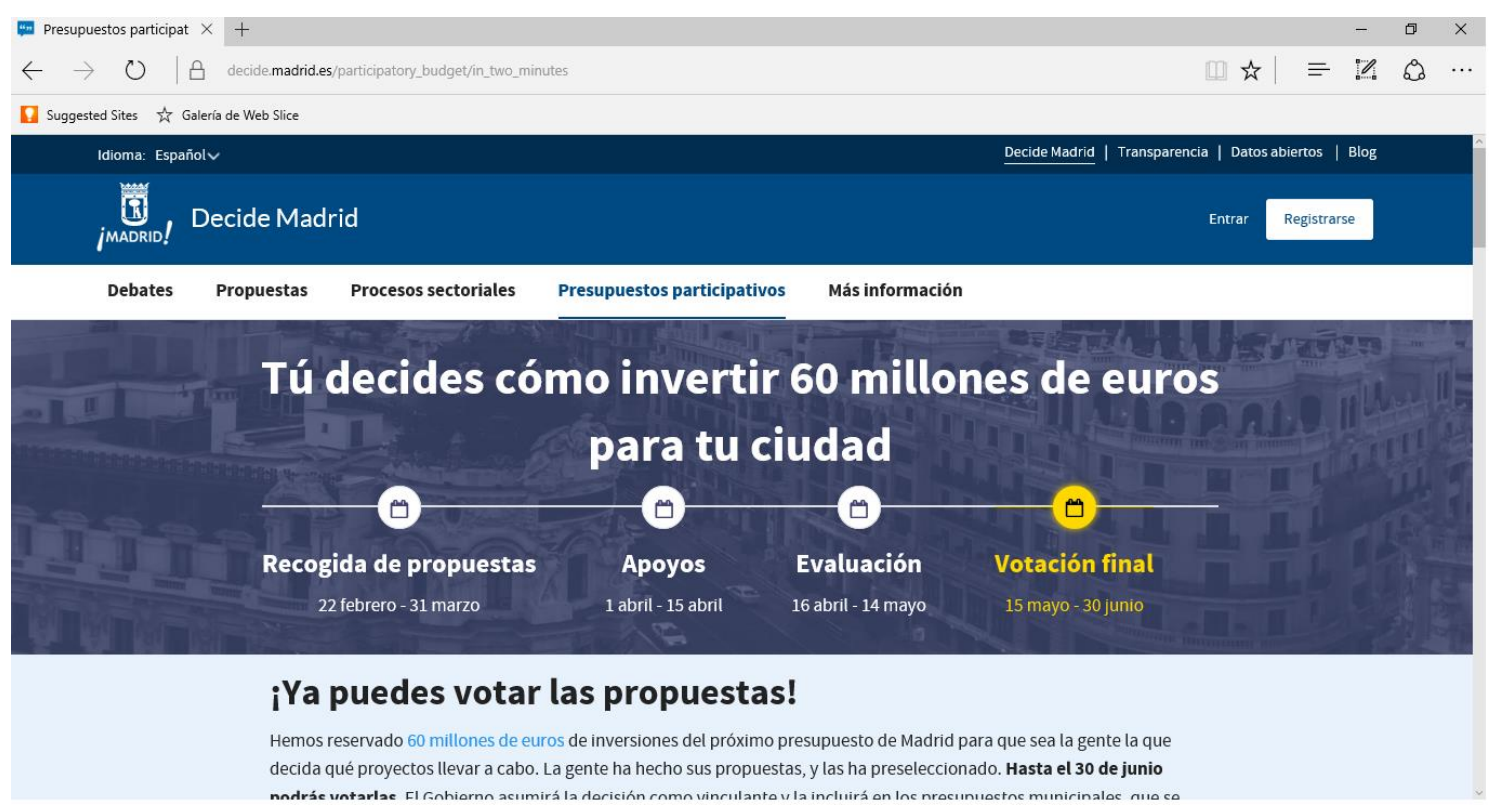

Hay también un apartado de preguntas frecuentes en los que intentan resolver las principales dudas que puedan tener los ciudadanos. Podía haber sido interesante, especialmente en esta primera ocasión, haber utilizado un cuadro de mensajes para plantear dudas, similar al utilizado en la web del presupuesto participativo de Paris.

Opinión, información, formación, derecho a expresarse, son en los procesos participativos tan importantes como la votación en sí y en este proyecto no se percibe que sea así. Igualando la e-participación a la e-votación se trivializa la posibilidad de la mejora de los procesos participativos mediante las TIC. Formas emancipadoras de utilización de las TIC exigen la implicación de los ciudadanos, que participen de un proceso activo, y su reconocimiento como actores en el debate político.

La ausencia de una deliberación de calidad es otra de las críticas que se puede realizar a este proceso, al ser considerado por los expertos un aspecto fundamental de los presupuestos participativos. Las herramientas de deliberación online están ya disponibles y son de mucha calidad, como se puede ver en el caso de Reykjavík, por lo que el problema no es que estas herramientas no existan sino que no se han utilizado, las razones las desconocemos. Estas herramientas de deliberación tienen que tener un interface de fácil lectura e entendimiento por

R.G. Secr., GESEC, São Paulo, v. 9, n. 1, p 265-292, jan./abr. 2018. 
Nebot, C. P. (2018).

parte de los individuos comunes que no cuentan con la expertise tecnológica. Alfaro, Gómez, Lavin y Molero (2010) hacen hincapié en que el presupuesto participativo online no debe reducirse a una pequeña plataforma y metodología de votaciones, sino ampliarse a las herramientas de negociación y de apoyo grupal para la toma de decisiones consensuada bajo publicación y con el método de incremento equilibrado.

Quizá muchos de los problemas que se han planteado se produzcan porque los PP online son elaborados por gestores públicos que no tienen el conocimiento especializado (expertise) de las redes digitales y de su mejor forma de utilización, pero creemos que no es el caso de la experiencia de Madrid pues el concejal responsable conoce perfectamente ese mundo. También puede ocurrir lo contrario, es decir que el gestor público sea un experto en TIC pero no tenga conocimientos sobre presupuesto participativo, algo que si creemos que se puede dar en esta experiencia. En la entrevista que tuvimos con el Director General, responsable de la gestión del proceso, éste nos manifestó que ni él ni las personas que trabajaban con él tenían conocimientos de participación ciudadana y menos de presupuesto participativo.

Otra cuestión importante que no podemos olvidar es la de la exclusión digital considerada por muchos expertos como una de las mayores limitaciones para la participación en el Presupuesto Participativo online. Como podemos ver en la Tabla 8, casi la mitad de los participantes (48\%) tienen entre 30 y 44 años y si ampliamos a 49 años supone el $60 \%$ de los participantes. En cambio, a partir de los 55 años va descendiendo la participación, cuando curiosamente esa franja de edad es la que más participa en los presupuestos participativos presenciales.

Sería interesante que el Servicio de Inclusión, Neutralidad y Privacidad, creado por este gobierno, que ha puesto en marcha una Mesa de Inclusión con técnicos municipales y asociaciones para trabajar con colectivos en situación de exclusión y al que se le ha encargado diseñar mecanismos especiales para que puedan participar dichos colectivos en este proceso, trabaje también para romper la brecha digital. También existen cuestiones socio-tecnológicas que necesitarían abordarse, referidas, principalmente, a la confianza de la ciudadanía en las tareas electrónicas (Lavin et al., 2014: 172). 
Tabla 8.- Participación por grupos de edad

\begin{tabular}{|c|c|c|}
\hline EDAD & TOTAL & PORCENTAJE \% \\
\hline $\mathbf{1 6 - 1 9}$ & 754 & 1,67 \\
\hline $\mathbf{2 0 - 2 4}$ & 2.393 & 5,28 \\
\hline $\mathbf{2 5 - 2 9}$ & 3.467 & 7,66 \\
\hline $\mathbf{3 0 - 3 4}$ & 6.242 & 13,79 \\
\hline $\mathbf{3 5 - 3 9}$ & 8.082 & 17,85 \\
\hline $\mathbf{4 0 - 4 4}$ & 7.544 & 16,66 \\
\hline $\mathbf{4 5 - 4 9}$ & 5.282 & 11,66 \\
\hline $\mathbf{5 0 - 5 4}$ & 4.098 & 9,05 \\
\hline $\mathbf{5 5 - 5 9}$ & 3.086 & 6,82 \\
\hline $\mathbf{6 0 - 6 4}$ & 2.108 & 4,66 \\
\hline $\mathbf{6 5 - 6 9}$ & 1.214 & 2,68 \\
\hline Más de 70 & 1.011 & 2,23 \\
\hline
\end{tabular}

Fuente: Ayuntamiento de Madrid.

La tecnología utilizada no ha servido para mejorar la información sobre el presupuesto ni para que más ciudadanos conocieran la experiencia que se había puesto en marcha. Y aunque ha conseguido que participe una franja de edad que no suele hacerlo, ha excluido a los que si suelen hacerlo en las experiencias presenciales. Por otro lado, no se ha creado un espacio de deliberación online en el proceso, un aspecto considerado fundamental por los expertos en los presupuestos participativos.

\section{Conclusiones}

El paso más difícil en un proyecto de PP sea presencial u online es ponerlo en marcha y que las adaptaciones y mejoras se vayan realizando durante el proceso. En cuanto a las razones de los fracasos y las interrupciones son similares en los dos modelos de PP: falta de voluntad política, de cultura participativa y un diseño institucional inadecuado.

Es por ello muy importante realizar una evaluación objetiva y amplia, tanto del diseño como del funcionamiento, al final de cada período y más aún los primeros años de funcionamiento. No hay que tener miedo de realizar cambios si se considera que con ello el proceso puede mejorar, no hay que entenderlo como un fracaso sino al contrario como un progreso. Tampoco hay que olvidar que uno de los valores del proyecto de presupuesto participativo es el dinamismo. 
Nebot, C. P. (2018).

Sin olvidar tampoco su carácter didáctico, para ello es necesario facilitar más información y explicar cada una de los pasos que se van realizando. La cultura de las explicaciones parece que sigue siendo algo ajeno a nuestra cultura política: las autoridades parecen poco dispuestas a dar explicaciones públicas cuando las propuestas se rechazan o sufren algún cambio.

También hay que tener en cuenta que una participación meramente agregativa, aunque ampliada y masiva, no es suficiente para convertir en idóneos los procesos de deliberación pública, ni tampoco para fortalecer la ciudadanía cívica. Cualquier experimento democrático participativo, sea presencial u online, debe suponer una acción político-social contundente, sea por medio de la búsqueda de información, el debate y discusión de las grandes cuestiones públicas; sea decidiendo a través del voto, acompañando y fiscalizando la implementación de lo que fue decidido o sea exigiendo la debida accountability por parte del poder público.

Los problemas con los que nos encontramos en los presupuestos participativos que hacen uso de las TIC en su metodología, sin embargo, no son de tecnología sino de cultura política, de desarrollo político. Tenemos mucha tecnología, aunque aún puede haber más y mejor, pero poca democracia, pues la tecnología que se podría usar para conseguir una democracia de mayor calidad no es utilizada para ello (Pinho y Winkler, 2007 y Pinho, 2008). Además corremos el riesgo de caer en un instrumentalismo con estas iniciativas de $e$ democracy ya que más participación no es necesariamente más democracia.

El primer proceso de presupuesto participativo de la ciudad de Madrid ha tenido muchas sombras: no ha existido una adecuada política de comunicación, la información ha sido escasa, no se ha facilitado ninguna información sobre el presupuesto municipal, ha habido poca transparencia, la coordinación con la otra área responsable de la participación colectiva no ha funcionado, ha habido poco personal con conocimientos sobre el tema y la tecnología utilizada no ha sido la más adecuada.

Pero también ha habido luces: cuentan con un apoyo político mayoritario y es el primer año en que se pone en marcha. Lo que les permite corregir los errores cometidos y mejorar la experiencia.

Por último, no debemos olvidar que la participación, sea presencial u online, no es un valor democrático por ser un valor en sí mismo, sino únicamente en la medida en que puede producir algún beneficio a la comunidad política (Gomes, 2011). Saber que se debe hacer para producir ese beneficio a los ciudadanos y gestionarlo bien, deben ser los referentes de cualquier acción política. Esperamos que este trabajo pueda servir para motivar a la reflexión 
y si se considera conveniente para cambiar aquellas cosas que no han funcionado en este primer año.

\section{Referencias}

Alfaro, C.; Gómez, J.; Lavin, J. M. y Molero, J. J. (2010). A configurable architecture for eParticipatory budgeting support. JeDEM-eJournal of eDemocracy and Open Government, 2 (1): 39-45.

Avritzer, L. (2003). O orçamento participativo e a teoria democrática: um balanço crítico, en Leonardo Avritzer y Zander Navarro (eds.) A inovação democrática no Brasil: o orçamento participativo. São Paulo: Cortez.

Borba, J. y Lüchmann, L. (2007). Orçamento Participativo: análise das experiências desenvolvidas em Santa Catarina. Florianópolis: Insular.

Carlos, E. (2011). Associativismo e desenho institucional no orçamento participativo da região metropolitana do Espírito Santo, Ciências Sociais Unisinos, 47(2): 116-128.

Comisión Permanente Ordinaria de Participación Ciudadana, Transparencia y Gobierno Abierto (CPCTGA) del 17 de julio de 2015.

Comisión Permanente Ordinaria de Participación Ciudadana, Transparencia y Gobierno Abierto (CPCTGA) del 30 de junio de 2016.

Comisión Permanente Ordinaria de Participación Ciudadana, Transparencia y Gobierno Abierto (CPCTGA) del 29 de julio de 2016.

Ganuza, E. y Baiocchi, G. (2012). “The Power of Ambiguity: How Participatory Budgeting T ravels the Globe". Journal of Public Deliberation, 8 (2) artigo 8. 
Nebot, C. P. (2018).

Gomes, W. (2011). Participação Política Online: Questões e hipóteses de trabalho en Rousiley C. M. M.; Wilson G. y Francisco P. J. A. M. Internet e Participação política no Brasil. Porto Alegre: Sulina.

Horochovski, R., Rossi y Clemente, A. J. (2012). Democracia deliberativa e orçamento público: experiências de participação em Porto Alegre, Belo Horizonte, Recife e Curitiba, Revista Sociología Política, 20 (43): 127-157.

Lavin, J. M.; Álvarez, E. y Mayorga, F. (2014). Deliberación y participación: senderos electrónicos, Methaodos. Revista de Ciencias Sociales, 2 (2): 171-181.

Lüchmann, L. (2002). Possibilidades e limites da democracia deliberativa: a experiência do Orçamento Participativo de Porto Alegre. Tese (Doutorado em Ciências Sociais) Unicamp: Campinas.

Lüchmann, L. (2014). 25 anos de Orçamento Participativo: algumas reflexões analíticas. Politica \& Sociedade (online), 13: 167-197.

Pateman, C. (2012). Participatory democracy revisited. Perspectives on Politics, 10 (01): 719.

Pinho, J. A. G. (2008). Investigando portais de governo eletrônico de estados no Brasil: muita tecnologia, pouca democracia. Revista de Administração Pública. RAP - Rio de Janeiro 42 (3): 471-93, mayo/junio.

Pinho, J. A. G. y Winkler, I. (2007). Dabliu, Dabliu, Dabliu: Sociedade da Informação. Que informação?. Anais do Encontro da Associação Nacional de Pesquisa em Administração - ANPAD, Rio de Janeiro.

Pires, V. y Pineda, C. (2008). Presupuesto Participativo: una tipología para superar los límites de las definiciones demasiado amplias o restrictivas, Revista de Estudios de la Administración Local y Autonómica (REALA) 308: 206-244. 
Porto de Oliveira, O. (2013). Embaixadores da participação: a difusão internacional do orçamento participativo a partir do Brasil. Tese de doutorado. Programa de PósGraduação em Ciência Política, São Paulo, Universidade de São Paulo.

Romão, W. y Silva, F. A. S. (2016). Una vieja innovación democrática: la trayectoria del presupuesto participativo en Brasil, $8^{\circ}$ Congreso Consejo Europeo de Investigaciones Sociales en América Latina, organizado por el Instituto de Iberoamérica, Universidad de Salamanca, 28 de junio al 1 de julio.

Sampaio, R. C. (2014). Orçamentos Participativos digitais: um mapeamento mundial das experiências já realizadas e suas contribuições para e-participação e e-democracia. Tesis presentada en la Universal Federal de Bahia (Brasil).

Spada, P. (2014). The Adoption and Abandonment of Democratic Innovations: investigating the rise and decline of participatory Budgeting in Brazil. International Congress of the Latin American Studies Association, 32., 2014, Chicago. Proceedings... Chicago: LASA.

Wampler, B. (2003). Orçamento Participativo: uma explicação para amplas variações nos resultados, en Luciano Avritzer y Zander Navarro (eds.) A inovação democrática no Brasil: o orçamento participativo. São Paulo: Cortez.

Wampler, B. (2007). Participatory budgeting in Brazil: contestation, cooperation, and accountability. University Park: The Pennsylvania State University Press. 